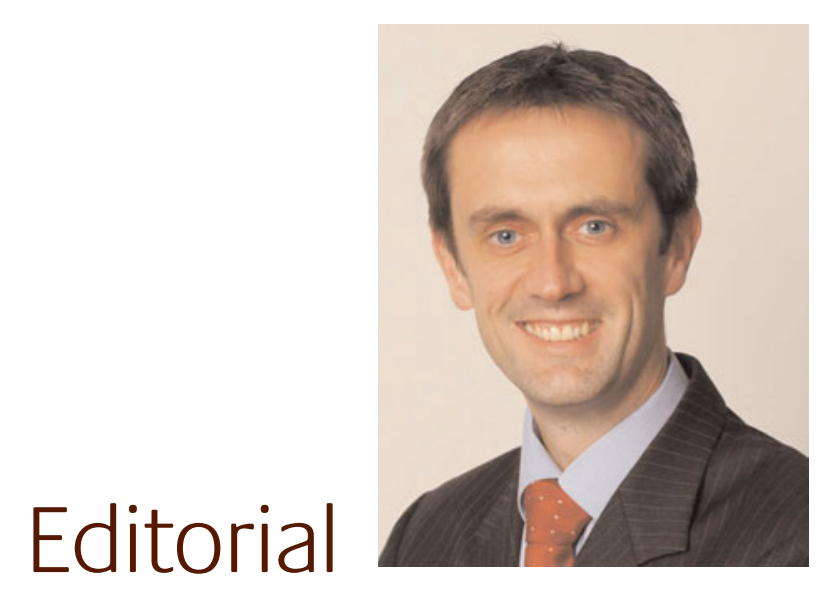

\section{A Growing Archive...}

Some dedicated, older readers of Gold Bulletin may be fortunate enough to own or have access to an extensive back catalogue of Gold Bulletin volumes. Of course, with our move to e-journal publishing from this issue, this collection of journals will no longer grow in hard copy form, which is, no doubt, of regret to some readers.

However, as the saying goes... 'as one door closes, so another one opens'...and one of the benefits of launching the new website for Gold Bulletin, www.goldbulletin.org is that we have been able to create a searchable web-based archive facility, into which we will be placing all the back issues of Gold Bulletin. With over 37 years of continuous journal publication, this is quite a task. All the older issues require conversion from hard copy form into electronic pdf files, so this expansion of the archive will obviously not happen overnight! Nonetheless, those of you who have visited the archive recently might have noticed that older back issues are now appearing. More issues will appear in the coming weeks, until eventually the full back catalogue is completed. All the papers will be free to download, resulting in what we believe will be one of the best technical journal archives on the internet and a unique resource for those working with gold!

\section{Gold Colloid}

In managing the expansion of the journal's archive on the new website, I have found it particularly interesting to familiarise myself with the range and number of excellent papers that have appeared in Gold Bulletin over the last four decades. The papers by John Turkevich published in 1985 (Gold Bull. 1985, 18, 86-91 and Gold Bull. 1985, 18, 125-131) are a classic example. Now twenty years old, these papers provided a first class review of the processing and properties of gold colloids that is still of great relevance and use today.

In recent weeks we have had a number of requests to obtain these papers, noticeably from individuals in the biomedical sector. This is not surprising, when one considers the unique properties of gold colloid are now being used in many applications in this sector, including pregnancy testing kits and most recently medical testing for HIV/AIDs.

As a result of the high demand for these papers, Parts I and II of John Turkevich's papers can now be downloaded from the Gold Bulletin archive for the benefit of all readers!

Enjoy this issue of Gold Bulletin and be sure to search the archive the next time you visit www.goldbulletin.org

Best regards

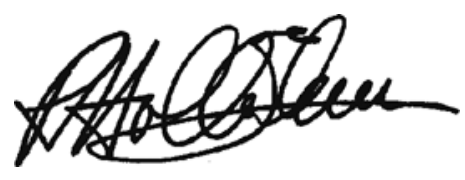

Richard Holliday

World Gold Council 